\title{
Efecto de la inversión pública en la infraestructura vial sobre el crecimiento de la economía peruana entre los años 2000-2016
}

\author{
Carlos Arturo Palacios Tovar* \\ Universidad Nacional Mayor de San Marcos \\ Recibido: 11 de abril del 2018 / Aprobado: 23 de mayo del 2018 \\ doi: 10.26439/ing.ind2018.n036.2454
}

RESUmen: En la presente investigación, se apreciará cómo el efecto de la inversión pública en infraestructura vial influye en el crecimiento del PBI en el Perú durante el periodo 2000-2016; asimismo, se determinará cuál es el elemento generador de competitividad entre las regiones del país.

Palabras clave: desarrollo económico / inversiones públicas / gasto público / producto bruto interno

\section{The effect of public investment in road infrastructure on the Peruvian economic growth between the years 2000 and 2016}

\begin{abstract}
AвSTRACT: The present research will show how public investment in road infrastructure influenced the gross domestic product (GDP) growth in Peru between the years 2000 and 2016. Likewise, it will determine which element generates competitiveness among the regions of the country.
\end{abstract}

Keywords: economic development / public investments / public expenditure / gross domestic product

* Correo electrónico: cpalaciostovar@gmail.com 


\section{INTRODUCCIÓN}

Durante la ejecución del trabajo de investigación, se comenzó analizando la problemática vial y su incidencia en el crecimiento económico del país en el periodo 2000-2016, y se evidenció que hubo un impacto positivo en la productividad de los diversos factores de manera eficiente.

En efecto, la existencia de más y mejor infraestructura, vinculada a la prestación de servicios de forma adecuada se traducirá necesariamente en una disminución de costos para la empresas, y se logrará por ello mejorar su productividad y por lo tanto hacerse extensivo en la competitividad del país (aumento del producto bruto interno), lo que va a estar relacionado directamente con el fomento de atraer nuevas inversiones a los diversos sectores productores del país.

Por ello la importancia del estudio de la inversión pública en infraestructura vial y cómo ella viene contribuyendo significativamente al crecimiento de la economía peruana en los diversos sectores productivos, los cuales determinan desde ya extensivamente una relación directamente positiva entre la inversión pública y el crecimiento del producto bruto interno del país.

\section{MARCO TEÓRICO}

La provisión deficiente de los servicios de infraestructura vial es uno de los aspectos más importantes de las políticas de desarrollo, especialmente en aquellos países que han orientado su crecimiento hacia el exterior. Para la gran mayoría de los especialistas, la ineficiencia de los servicios de infraestructura constituye un obstáculo de primer orden para la implementación eficaz de políticas de desarrollo y la obtención de tasas de crecimiento económico que superen los promedios internacionales. Por diversas razones de inclusión, los países requieren ampliar y modernizar su infraestructura básica de acuerdo con estándares tecnológicos internacionales, lograr niveles máximos de cobertura del territorio nacional y satisfacer con eficacia las necesidades de servicios de infraestructura de los agentes económicos y las personas.

Por otro lado, la adecuada disponibilidad de obras de infraestructura, sobre todo en el aspecto vial, así como la prestación eficiente de servicios generales, contribuirán a que un país o una región pueda desarrollar ventajas competitivas y alcanzar un mayor grado de especialización productiva. Asimismo, las redes de infraestructura también constituyen 
un elemento central de la integración del sistema económico y territorial de un país, y hacen posible las transacciones dentro de un espacio geográfico económico determinado y en el exterior. Por tal razón, tales redes constituyen un elemento vertebrador de la estructura económica de los países y de sus mercados, así como de los mecanismos concretos de articulación de las economías nacionales con la economía mundial.

Según la evidencia internacional, la infraestructura de servicios públicos constituye el soporte de toda actividad económica. De acuerdo con el Banco Mundial (1994), las tablas de insumo-producto de diversos países en vías de desarrollo muestran que, por ejemplo, la infraestructura de telecomunicaciones y de energía eléctrica es empleada en los procesos de producción de casi todos los sectores y que el transporte es un insumo esencial para la comercialización de los productos. El sector privado en estos países exige servicios de infraestructura vial no solo para su consumo directo, sino también para aumentar su productividad mediante la reducción del esfuerzo y del tiempo que son necesarios para la producción y para la distribución de sus bienes y servicios dentro del circuito económico.

Por tal razón, la existencia de infraestructura vial en una economía genera una serie de externalidades para el desarrollo de las actividades privadas, dado que se constituye en un conjunto de activo público que generalmente afecta las decisiones de producción y de consumo de las empresas y de los hogares sin que medien para ello mecanismos de mercado. Por ejemplo, los negocios privados requieren de una red de carreteras y caminos en buen estado para desarrollarse satisfactoriamente. Las actividades privadas no se llevarían a cabo adecuadamente si es que la infraestructura vial no fuera provista, ya sea por el sector público y/o por el sector privado de manera eficiente, lo que evita el desperdicio de los recursos escasos.

En teoría, el incremento de la capacidad productiva potencial de una economía puede ocurrir a través del suministro de una mayor cantidad de infraestructura vial, lo que provoca que la estructura de costos de las empresas se vean afectados a consecuencia de las externalidades generadas por la aglomeración y la concentración de la infraestructura existente en zonas donde la demanda efectiva por servicios de transporte terrestre es importante (Albala-Bertrand y Mamatzakis, 2001; Fujita y Krugman, 1999). Es así que cuando una empresa privada utiliza la infraestructura vial, la productividad de dicha forma crece porque esta combinación provoca la reducción de sus costos unitarios. 
Por esta razón, la expansión de la infraestructura vial puede elevar el rendimiento interno de los proyectos que las empresas privadas llevan a cabo, lo que indudablemente incrementa la rentabilidad de las inversiones del sector privado (Reinikka y Svensson, 1999). Esta situación genera mayores incentivos para que el sector privado realice inversiones, lo que llevará a un incremento en el crecimiento económico.

Por otro lado, puede existir un vínculo positivo entre la inversión en infraestructura vial y el crecimiento debido a que la expansión de la infraestructura puede estimular la inversión privada en capital, sea nacional o extranjera, al generar las condiciones apropiadas de mercado para el desarrollo de los negocios y de las empresas privadas en un país. De acuerdo con Vásquez (2003), existen principalmente dos canales por los que se establecen vínculos entre el crecimiento y la infraestructura.

- Canal 1: La expansión de la infraestructura vial genera aumentos en la capacidad productiva potencial de una economía.

- Canal 2: El incremento de la infraestructura vial genera cambios favorables en los precios relativos, ya que genera condiciones para el funcionamiento de los mercados de manera más eficiente.

Sin embargo, si tanto el sector privado como el sector que provee la infraestructura vial (que puede estar bajo la administración estatal o bajo régimen privado, vía una concesión administrativa o una privatización) compiten por un mismo conjunto de recursos, un incremento en el gasto en infraestructura podría generar un efecto negativo en el corto o en el largo plazo vía la reducción de los niveles de inversión privada al provocar un efecto conocido como crowding out (es decir, la inversión en infraestructura agota los recursos disponibles y desplaza a la inversión en capital privado). Por el contrario, si la competencia por el conjunto de recursos no es intensiva, entonces el efecto crowding in (es decir, existen incentivos para la inversión privada ante las mejoras en eficiencia) de la expansión de la infraestructura vial es superior al efecto crowding out.

La posibilidad de ocurrencia de esto efectos sugiere la idea de que la relación entre la expansión de la infraestructura vial y el crecimiento es una de tipo no lineal. Ello implica que, para determinado valor del umbral de la razón capital en infraestructura y capital privado, la relación entre infraestructura y crecimiento se revierte. De esta forma, para niveles bajos de dotación de infraestructura vial en relación con el capital privado, la relación entre el incremento de la infraestructura vial y el crecimiento es positivo (efecto crowding in), mientras que, para nive- 
les de infraestructura en relación con el capital privado por encima del umbral, la relación se revierte a un tipo negativo (efecto crowding out).

La evidencia internacional muestra que existe una fuerte asociación entre la disponibilidad de cierta infraestructura (como las instalaciones de telecomunicaciones, la capacidad de generación eléctrica, las carreteras pavimentadas, los ferrocarriles, etc.) y el producto bruto interno per cápita en los países en desarrollo. Datos correspondientes a 1990 indican que, mientras que el capital total de infraestructura aumenta en $1 \%$ con cada incremento de $1 \%$ del PBI per cápita, el acceso de las familias y de las empresas a las carreteras pavimentadas, a la energía eléctrica y a las telecomunicaciones aumentan en 0,8 \%, 1,5\% y 1,7 \%, respectivamente (Banco Mundial, 1994). Entonces, puede decirse que las inversiones en infraestructura vial en los países en desarrollo son necesarias para generar aumentos sostenibles del crecimiento económico a través de la expansión de la capacidad productiva de un país, la mejora de la eficiencia económica y la transparencia de los precios relativos.

Barro (1990), basado en el trabajo de Romer (1986), utiliza un modelo de crecimiento endógeno para sostener que el gasto público en infraestructura tiene un efecto causal positivo directo sobre el crecimiento económico y el bienestar. Luego de estas investigaciones iniciales, numerosos estudios, con información de países a nivel nacional e internacional, han encontrado evidencia sobre lo importante que es la infraestructura para estimular el crecimiento económico. Por ejemplo, al utilizar datos de Servicios Cruzados, Eastert y Rebelo (1993) y Yamarik (2000), encontraron un efecto positivo de la inversión en transporte sobre el crecimiento. Canning Fay y Perotti (1994) hallaron un impacto positivo de la infraestructura telefónica sobre el crecimiento económico para diversos países de Latinoamérica, y Sánchez Robles (1998) encontró efectos positivos significativos de la infraestructura de generación eléctrica y de teléfonos sobre el crecimiento.

Canning (1999), mediante el uso de datos de panel para varios países, encontró que existen indicios para sostener que la infraestructura tiene repercusiones considerables sobre el crecimiento. Bernand y García (1997) realizaron una evaluación de los impactos de la infraestructura sobre el crecimiento económico para el caso de algunos países desarrollados y del tercer mundo.

Mediante un simple modelo de crecimiento endógeno, los autores hallaron que la infraestructura tiene repercusiones considerables sobre 
el crecimiento siempre y cuando existan procesos de financiamiento adecuado para tales inversiones. En contraste, Holtz-Eaking y Schwartz (1994), y Garcia-Mila, McGuire y Porter (1996) sugieren que hay poca evidencia de un efecto significativo de la infraestructura sobre el crecimiento del producto. Para llevar a cabo su estado, los autores emplearon datos de panel a nivel de Estados Federales para el caso estadounidense y un modelo econométrico de datos de panel que incorporan efectos fijos.

Para el caso de Latinoamérica, existen aún pocos estudios a nivel agregado relacionados con el tema de infraestructura y de crecimiento. Puede mencionarse un estado realizado por Albaba-Bertrand y Mamatzakis (2001) para el caso chileno que evalúa el efecto de la infraestructura de servicios públicos sobre la estructura de costos y la productividad de la economía chilena antes y después de las reformas neoliberales. A través de una estimación de una función de costos translogarítmica, ambos encontraron que la infraestructura ha provocado incrementos en la productividad significativa durante el periodo ex post a las reformas.

En el caso peruano, Uranga y Aparicio (2012) resaltan en su estudio la importancia de la infraestructura para el crecimiento económico al efectuar una estimación econométrica a fin de recoger la relación entre ambas variables en el caso peruano, y utilizar un panel de datos con información para las 24 regiones del Perú correspondiente al periodo 1980-2009 bajo distintos estimadores. Los resultados econométricos obtenidos confirman que la infraestructura de servicios públicos (carreteras, electricidad y telecomunicaciones) resulta relevantes para las diferencias transitorias en el producto regional, de acuerdo con las teorías neoclásicas de crecimiento exógeno. Por otra parte, se encuentra evidencia que respalda la presencia de diferencias significativas en las repercusiones de las infraestructuras en el producto per cápita de cada región.

\section{METODOLOGÍA}

El presente artículo corresponde a una investigación aplicada que junta los conocimientos sobre teoría económica e inversiones públicas.

La investigación aplicada, llamada también constructiva o utilitaria, se caracteriza por su interés en la aplicación de los conocimientos teóricos a determinada situación concreta y las consecuencias prácticas que de ella se deriven. Busca conocer para hacer, para actuar para construir, para modificar: le preocupa la aplicación inmediata sobre una 
realidad circunstancial antes que el desarrollo de un conocimiento de valor universal.

La unidad de análisis en el presente estudio es un dato anual del país y la muestra del periodo 2000-2016 con datos anuales por departamentos.

\subsection{Técnicas de recolección de datos}

Se utilizará información secundaria proporcionada por el Ministerio de Economía y Finanzas, Transparencia Económica, el Instituto Nacional de Estadística e Informática y documentos relacionados con el tema de investigación.

Los datos fueron recolectados de las bases de:

- Instituto Nacional de Estadística e Informática del Perú.

- Banco Central de Reserva del Perú.

- Empresa Cuanto.

- Informes Gubernamentales.

- Investigaciones relacionadas con el tema.

\section{ANÁLISIS E INTERPRETACIÓN DE LA INFORMACIÓN}

\section{La metodología de análisis e investigación}

El método a utilizarse en la presente investigación será el MÉTODO CIENTÍFICO con los procedimientos DEDUCTIVO E INDUCTIVO y el método histórico. El primero consiste en analizar de manera general la inversión pública en infraestructura vial. Asimismo, este procedimiento nos permite partir de un contexto general a algo particular y luego centrarnos en el tema de los impactos del crecimiento económico sobre la inversión pública en infraestructura. El segundo procedimiento nos permite arribar a conclusiones generales al partir de hechos particulares. En esta última parte entra el método histórico, porque el procedimiento es esencialmente inductivo.

Para nuestro estudio, utilizaremos un modelo econométrico, el cual se trabajó en el Microsoft Excel para la base de datos, y el programa Eviews-6 para el modelo econométrico propiamente. 
Modelo por establecerse:

$$
\mathrm{TC}=80+81 \cdot \mathrm{IPT}+\text { 62.GPT }+ \text { 63.PES + 84 } \cdot \mathrm{PAE}+\mathrm{e}
$$

Dónde:

TC: Tasa de crecimiento económico.

IPT: Inversión pública en transportes.

GPT: Gasto público total.

PES: Población con educación superior.

PAE: Población adecuadamente empleada.

\section{RESULTADOS}

De los resultados y las pruebas de hipótesis, podemos afirmar que las variables que aportan a la explicación de la variable crecimiento económico.

\section{Para la variable GPT: gasto público total}

Se pudo probar el grado de influencia que tiene la variable independiente (gasto público total) sobre la variable dependiente (tasa de crecimiento económico). Por lo tanto, esta variable aporta significativamente al modelo con un nivel de confianza del $95 \%$.

\section{Para la variable IPT: inversión pública en transportes}

Se pudo probar el grado de influencia que tiene la variable independiente (inversión pública en transportes) sobre la variable dependiente (tasa de crecimiento económico). Por lo tanto, esta variable aporta significativamente al modelo con un nivel de confianza del $95 \%$.

\section{Para la variable PAE: población adecuadamente empleada}

Se pudo probar el grado de influencia que tiene la variable independiente (población adecuadamente empleada) sobre la variable dependiente (tasa de crecimiento económico). Por lo tanto, esta variable aporta significativamente al modelo con un nivel de confianza del $95 \%$.

\section{Para la variable PES: población con educación superior}

No se pudo probar el grado de influencia que tiene la variable independiente (población con educación superior), sobre la variable dependiente (tasa de crecimiento económico). Por lo tanto, esta variable no aporta significativamente al modelo con un nivel de confianza del $95 \%$. 
Tomando en cuenta los valores beta (Bi) que tiene el modelo de regresión final estimada, se presenta de la siguiente forma:

$$
\mathrm{TC}=-20.32845-0.698905 \mathrm{TC}(-1)+8.17 \mathrm{E}-06 \mathrm{GPT}+-6.36 \mathrm{E}-13 \mathrm{GPT} 2
$$

-7.97E-05 IPT + 7.88E-11 IPT2 + 6.87E-08 PAE + 2.08E-12 PAE2 + 1.07E-07PES $-3.38 \mathrm{E}-16 \mathrm{PES} 2$

Tabla 1

Prueba de residuales del modelo

\begin{tabular}{|c|c|c|c|c|c|}
\hline obs & Actual & Fitted & Residual & & Residual Plot \\
\hline 2000 & 0,27 & 0,1027452 & 0.1672547 & 1 & . $1 *$ \\
\hline 2001 & 0,06 & 0,267166 & -0.2071669 & | & $.{ }^{*} \mid$ \\
\hline 2002 & 0,55 & 0,691852 & -0.1418520 & | & ${ }^{*} \mid$ \\
\hline 2003 & 0,42 & 0,599530 & -0.1795308 & I & $.{ }^{*} \quad \mid$ \\
\hline 2004 & 0,5 & 0,602897 & -0.1028973 & I & ${ }^{*} 1$ \\
\hline 2005 & 0,63 & 0,5618567 & 0.0681432 & I & . $\quad 1^{*}$ \\
\hline 2006 & 0,75 & 0,5824081 & 0.1675918 & 1 &.$\quad 1^{*}$. \\
\hline 2007 & 0,85 & 0,6360614 & 0.2139385 & I & . $\left.\right|^{*}$. \\
\hline 2008 & 0,91 & 0,6967938 & 0.2132061 & I & . $1{ }^{*}$. \\
\hline 2009 & 0,1 & 0,5721096 & -0.4721096 & $1^{*}$ & .1 \\
\hline 2010 & 0,85 & 0,5017930 & 0.3482069 & | & .1 \\
\hline 2011 & 0,65 & 0,4721531 & 0.1778468 & I & . $1{ }^{*}$. \\
\hline 2012 & 0,6 & 0,5900705 & 0.0099294 & I & . * \\
\hline 2013 & 0,58 & 0,5827785 & -0.0027785 & I & * \\
\hline 2014 & 0,24 & 0,4204846 & -0.1804846 & I &.${ }^{*} 1$ \\
\hline 2015 & 0,33 & 0,3731340 & -0.0431340 & I & . ${ }^{*}$ \\
\hline
\end{tabular}

Elaboración propia

Mediante la prueba de residuales, el modelo no presenta rezagos y se puede afirmar que el modelo es estable para predecir el comportamiento de la variable tasa de crecimiento. 


\begin{tabular}{|c|c|c|c|c|c|c|c|c|}
\hline \multicolumn{9}{|c|}{ Sample: 20002016} \\
\hline \multicolumn{9}{|c|}{ Included observations: 17} \\
\hline \multicolumn{2}{|c|}{ Autocorrelation } & \multicolumn{2}{|c|}{ Partial Correlation } & & $A C$ & PAC & Q-Stat & Prob \\
\hline 1 & 1 & 1 & 1 & 1 & $-0,100$ & $-0,100$ & 0,2021 & 0,653 \\
\hline I & 1 & 1 & 1 & 2 & $-0,081$ & $-0,092$ & 0,3423 & 0,843 \\
\hline I & I & I & I & 3 & $-0,091$ & $-0,111$ & 0,5323 & 0,912 \\
\hline । & I & $\square$ & 1 & 4 & $-0,177$ & $-0,214$ & 1,3130 & 0,859 \\
\hline I & 1 & 1 & I & 5 & 0,113 & 0,047 & 1,6550 & 0,895 \\
\hline I & 1 & 1 & I & 6 & $-0,023$ & $-0,057$ & 1,6700 & 0,947 \\
\hline I & I & Б & I & 7 & $-0,079$ & $-0,123$ & 1,8706 & 0,967 \\
\hline I & I & 1 & I & 8 & 0,006 & $-0,051$ & 1,8717 & 0,985 \\
\hline 1 & 1 & $1 \square$ & 1 & 9 & $-0,306$ & $-0,346$ & 5,6615 & 0,773 \\
\hline I & 1 & & I & 10 & 0,045 & $-0,124$ & 5,7546 & 0,835 \\
\hline । & 1 & 1 & I & 11 & 0,093 & $-0,058$ & 6,2232 & 0,858 \\
\hline I & I & 1 & 1 & 12 & 0,059 & $-0,051$ & 6,4459 & 0,892 \\
\hline
\end{tabular}

Figura 1. Prueba de correlograma de residuales del modelo Elaboración propia

Mediante la prueba de residuales, el modelo no presenta rezagos y se puede afirmar que el modelo es estable para predecir el comportamiento de la variable tasa de crecimiento.

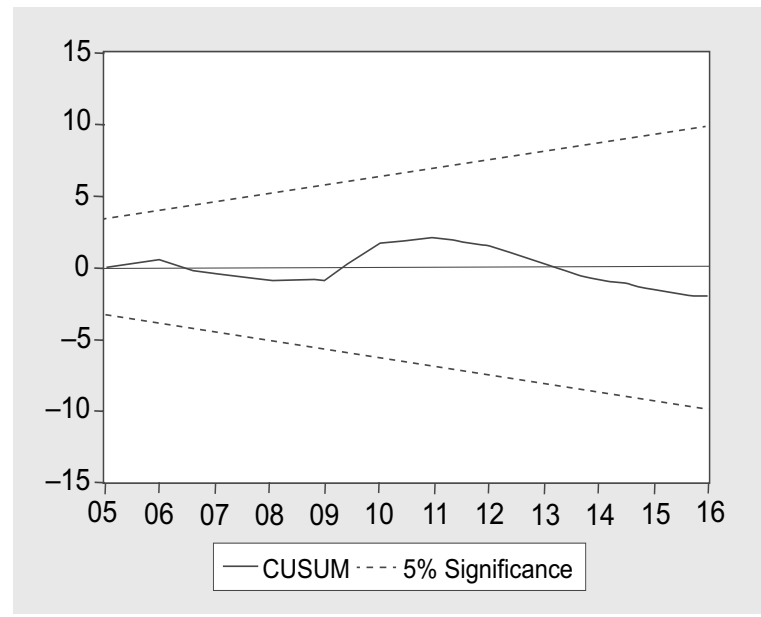

Figura 2. Prueba de estabilidad del modelo Cusum Elaboración propia

Mediante la prueba de Cusum, podemos afirmar que el modelo es estable para predecir el comportamiento de la variable tasa de crecimiento. 


\section{CONCLUSIONES}

Se puede afirmar que en la presente investigación se ha podido obtener lo siguiente:

1. Estadísticamente se ha podido probar que la inversión pública en infraestructura vial ha contribuido positiva y significativamente sobre el crecimiento de la economía peruana.

2. El sistema de transporte vial es un generador inmediato de movimiento económico, lo que origina casi siempre índices positivos de crecimiento económico sostenido en el país, con lo que se reflejan mejoras directas en la reducción de tiempos de transporte para el trabajo, comercio cualquiera fuese su naturaleza, al reducir los costos de los productos que se comercializan y abrir además nuevas opciones de mercado, con el objeto de integrar exitosamente al Perú en la economía global del siglo XXI.

3. Estadísticamente, se ha podido probar además que el crecimiento de la infraestructura vial ha contribuido positiva y significativamente sobre la actividad económica de los sectores productivos.

4. Nuestro país tiene mucho por construir en materia de carreteras, siquiera para intentar asemejarse a Brasil, cuya densidad vial es 66,4 $\%$ mayor que la de nuestro país, o para contar con el porcentaje de carreteras asfaltadas respecto del total de kilómetros de carreteras de Chile, que es 2,6 veces mayor que la de nuestro país.

5. Estadísticamente, se ha podido probar que la infraestructura vial ha contribuido positiva y significativamente en el poceso de crecimiento regional en el Perú.

6. Estadísticamente, se ha podido probar que la infraestructura vial es un elemento generador de igualdad económica a nivel regional que contribuye positiva y significativamente.

7. Es importante converger hacia una agenda que comprenda un conjunto de políticas integrales y articuladas que apunten en una misma dirección y que se constituyan en eslabones de un crecimiento sostenido al disponer la agenda de proyectos junto con los cronogramas de ejecución a mediano y largo plazo, responsables de la ejecución y el monitoreo permanente.

8. El crecimiento económico se da al favorecer la incorporación de nuevos sectores públicos al incrementar y multiplicar un crecimiento y un desarrollo económico sostenibles, debido a la inversión pública en infraestructura vial. Por lo tanto, extensivamente se considera que 
existe una relación directa entre inversión pública y crecimiento del producto bruto interno.

9. El déficit de infraestructura vial puede ser visto como una oportunidad estratégica para la inversión privada al contar con la debida estabilidad jurídica en el marco de la normatividad vial vigente.

\section{RECOMENDACIONES}

1. Al probar que existe una relación directa entre inversión, competitividad y crecimiento del producto bruto interno en el país, se recomienda probarlo en las regiones del país, con mayores niveles de inversión, para medir si realmente son los más competitivos y, por ello, tienen tendencias de mayores niveles de inversión, lo cual consecuentemente se espera que genere un mayor crecimiento económico.

2. Asimismo, se recomienda probarlo en las regiones del país, con menores niveles de inversión, para medir si realmente son los menos competitivos y, por ello, tienen tendencias de menores niveles de inversión, lo cual consecuentemente se esperará que genere un menor crecimiento económico en la región dada.

3. Es de importancia estratégica la integración regional y territorial de nuestra economía, ya que se ha mencionado que las redes viales son importantes para unir las zonas productivas con los mercados locales, regionales, nacionales e internacionales, además de una red vial adecuada que permitirá fomentar la industria sin chimenea en el país.

4. Reformular el canon minero de tal modo que se redistribuya homogéneamente en todo el país, y no en exceso en pueblos que derrocharon indebidamente en proyectos innecesarios dichos recursos financieros.

5. Se recomendaría que se realice un estudio similar para encontrar un efecto positivo de la población con estudios superiores y la población adecuadamente ocupada sobre el crecimiento económico de una región, en un periodo largo a periodos mayores a 20 años de estudió.

6. La gestión de conservación de carreteras de la red vial nacional deberá reimpulsarse con la previsión de llegar a niveles altos de deterioro que generen elevados costos de rehabilitación y/o reconstrucción.

7. Los proyectos viales deben ser considerados de interés nacional, y su aprobación debe ser acelerada a fin de dinamizar el desarrollo territorial de los corredores económicos.

8. La inversión pública en el desarrollo vial del Perú generará un impacto de costo-beneficio favorable a la población peruana. 


\section{REFERENCIAS}

Banco Central de Reserva del Perú (2011). Memoria 2010. Lima: Autor.

Banco Mundial. (2011). Perspectivas de la Economía Mundial.

Desarrollo económico. (2017). En Enciclopedia de características (revisado en el 2017). Recuperado de http://www.caracteristicas. co/desarrollo-economico/\#ixzz4duEV4iiVDesde

Esfahani, H. S. y Ramírez, M. T. (2003). Institutions, Infrastructure, And Economic Growth. Journal of development Economics, 35(1), 443-477.

Garay. C. (2012). Crecimiento Con Inclusión-Ruta Al Primer Mundo: Cuatro Desafíos Del Quinquenio 2011-2016. Lima: Instituto Economía Desarrollo Empresarial de la Cámara de Comercio de Lima.

Hernández, J. (2010). Inversión pública y crecimiento económico: Hacia una nueva perspectiva de la función del gobierno (tesis doctoral). Universidad Autónoma Metropolitana de México.

Instituto Peruano de Economía [IPE]. (2017). Lima. Información Económica. Indicador Compuesto de Actividad Económica ICAE Consolidado 2016.

Loayza N. (2008). Causas y Consecuencias de la Informalidad en el Perú. Revista de Estudios Económicos. Estudios Económicos. BCRP, (15), 45-64.

Lucas R. (1988). In The Mechanics Of Economic Development Government Spending in a Simple Model Endogenous Growth. The Journal Of Political Economy, 98(5), 103-125.

Martínez, M. (2010). Ciencia y arte en la Metodología Cualitativa. Caracas: Editorial Trillas.

Ramírez, T. (2007). Cómo hacer un Proyecto de Investigación. Caracas: Editorial Panapo.

Rozas, P. y Sánchez, R. (2004). Desarrollo de Infraestructura y Crecimiento Económico: Revisión Conceptual. Recursos Naturales e Infraestructura. CEPAL. Santiago: Editorial Naciones Unidas.

Servén, L. y Calderon, C. (2004). The Effects Of Infrastructure Development on Growth and Income Distribution Dataset. 
Recuperado de https://openknowledge.worldbank.org/bitstream/ handle/10986/14136/WPS3400.pdf?sequence=1\&isAllowed=y

Zegarra, L. F. (2010). Competitividad Infraestructura y Desarrollo Regional. En Opciones de Política Económica en el Perú: 20112015. Lima: Fondo Editorial de la Pontificia Universidad Católica del Perú. 